\title{
Musgos (Bryophyta) da microrregião do Salgado Paraense e SuA UTILIZAÇ̃̃o COMO POSSÍVEIS INDICADORES DE AMBIENTES PERTURBADOS ${ }^{1}$
}

\author{
Rita de Cássia Pereira dos Santos ${ }^{2,3}$ \& Regina Célia Lobato Lisboa ${ }^{2}$
}

\begin{abstract}
Resumo
(Musgos (Bryophyta) da microrregião do Salgado Paraense e sua utilização como possíveis indicadores de ambientes perturbados) Foi realizado um estudo dos musgos ocorrentes em diferentes ecossistemas da microrregião do Salgado Paraense, visando determinar a riqueza, registrar os tipos de ecossistemas e substratos de ocorrência das espécies, identificar algumas destas como indicadoras de ambientes perturbados e ampliar o conhecimento da brioflora do estado do Pará. Foram registradas 38 espécies de musgos, distribuídas em 21 gêneros e 11 famílias. A riqueza de espécies foi relativamente baixa, levando-se em consideração a grande quantidade de amostras analisadas (558). Calymperes afzelii, C. erosum, C. lonchophyllum, C. palisotii, Octoblepharum albidum var. albidum, O. pulvinatum, Sematophyllum subsimplex, Splacnobryum obtusum e Taxithelium planum foram as que apresentaram melhor adaptação a condições adversas. Sugere-se que estas espécies podem ser potencialmente utilizadas como indicadoras de ambientes perturbados. Entretanto, todas as espécies ocorrentes nos oito municípios desta microrregião estão adaptadas a um complexo de fatores ambientais e encontram as condições necessárias e favoráveis à sua manutenção e reprodução.
\end{abstract}

Palavras-chave: briófitas, brioflora, Pará, ecossistemas, riqueza de espécies.

\section{Abstract}

(Mosses (Bryophyta) of the micro-region of Salgado Paraense and its use as possible indicators of disturbed ambient) A study of the mosses in different ecosystems of the micro-region of the Salgado Paraense, northeastern State of Pará, was carried out. This study aimed to determine species richness, the ecosystem types and the substrates of occurrence of the species, as well as to identify species as indicators of disturbed environments and to increase to the knowledge of the bryoflora of Pará. This inventory recorded 38 moss species, distributed in 21 genera and 11 families. The species richness was relatively low, considering the large number of examined specimens (558). Calymperes afzelii, C. erosum, C. lonchophyllum, C. palisotii, Octoblepharum albidum var albidum, O. pulvinatum, Sematophyllum subsimplex, Splacnobryum obtusum and Taxithelium planum were better adapted to adverse conditions. Potentially, these species could be used as indicators of disturbed environments. However, all the species collected in the eight municipalities are adapted to a combination of environmental factors and find the necessary and favorable conditions for their maintenance and reproduction. Key words: Bryophytes, Bryoflora, ecossystems, Pará, species richness.

\section{INTRODUÇÃO}

As briófitas constituem o segundo maior grupo de plantas terrestres, depois das angiospermas (Buck \& Goffinet 2000). Pertencem a um grupo monofilético de três divisões de plantas criptogâmicas (Marchantiophyta, Anthocerotophyta e Bryophyta) consideradas as mais simples no Reino Plantae devido à ausência de sistema vascular e cutícula epidérmica, dentre outros caracteres (Shaw \& Goffinet 2000).

Estas plantas são importantes componentes da biomassa de muitos ecossistemas. Em alguns hábitats, a camada de briófitas assimila e estoca muito mais carbono que todo o caule das árvores, liberando para a atmosfera grande quantidade de oxigênio; auxiliam também no controle da erosão do solo e assoreamento dos rios; são indicadoras da qualidade do solo, condições de PH e nível de água. Algumas espécies de musgos indicam a presença de cálcio ou nutrientes na água, outras são indicadoras paleoecológicas, bioindicadoras e/ou biomonitoras em estudos de poluição ambiental, bem como de certos minérios, pois concentram facilmente o cobre e o enxofre (Frahm \& Kirchhoff 2002; Lisboa

Artigo recebido em 10/2007. Aceito para publicação em 04/2008.

${ }^{1}$ Parte da dissertação de Mestrado da primeira Autora, apresentada a Coordenação de Botânica do MPEG.

${ }^{2}$ Museu Paraense Emílio Goeldi, Coordenação de Botânica.

${ }^{3}$ Autor para correspondência: Museu Paraense Emílio Goeldi, Coordenação de Botânica, Laboratório de Briologia, C.P. 399, 66040-170, Belém, PA, Brasil. rcsantos@ museu-goeldi.br. 
\& Ilkiu-Borges 1995; Lisboa \& Ilkiu-Borges F. 1996; Sergio \& Bento-Pereira 1981; Zartman 2003). Rao (1982) estudou os efeitos e a relativa sensibilidade de várias espécies de briófitas para diferentes tipos de poluentes do ar, comprovando seu potencial como bioindicadores da poluição atmosférica. De acordo com Gradstein et al. (2001), as briófitas são sensíveis às mudanças da umidade ambiental e qualidade do ar e da água, constituindo-se assim, plantas indicadoras de pequenas transformações climáticas e das condições ambientais e, indiretamente, de distúrbios no ecossistema.

Até 100 anos atrás, a região nordeste do Pará possuía florestas altas de terra firme, matas de várzeas e igapós, campos e manguezais. Atualmente, pouco resta dessas matas originais, devido, principalmente, às atividades antrópicas, que têm contribuído para causar distúrbios significativos no meio ambiente, colocando em risco o ecossistema costeiro. A mesorregião do nordeste paraense possui uma paisagem inteiramente descaracterizada com mais de $90 \%$ da sua cobertura florestal original convertida em vegetação secundária (Wagner 1995). Salomão et al. (1996) apontam como principal característica desta região a formação de capoeiras novas e de baixo porte, ressaltando que restam hoje menos de $2 \%$ do um milhão de hectares de floresta densa do século passado.

Os trabalhos de coletas de briófitas nesta região iniciaram em 1994 e finalizaram em 2006. Até o presente, existia apenas um único trabalho publicado para essa área (Santos \& Lisboa 2003) resultado de um inventário dos musgos que ocorrem em diferentes tipos de vegetação de alguns municípios da Zona Bragantina, microrregião do Salgado e município de Viseu, todos localizados no nordeste paraense. Dentro desta visão é oportuna a realização deste trabalho, cujo objetivo é determinar a riqueza de espécies dos musgos da microrregião do Salgado, registrar os tipos de ecossistemas e substratos de ocorrência das espécies, identificar algumas destas espécies como possíveis indicadoras de ambientes perturbados e ampliar o conhecimento da Brioflora do estado do Pará.

\section{Material e Métodos Área de estudo}

A microrregião do Salgado Paraense faz parte da zona costeira e abrange uma área total de 5.812,70 $\mathrm{km}^{2}$. De acordo com SEPOF (2005), os 11 municípios limítrofes com o Oceano Atlântico, que compõem a Zona Fisiográfica do Salgado são: Colares, Curuçá, Magalhães Barata, Maracanã, Marapanim, Salinópolis, São Caetano de Odivelas, São João da Ponta, São João de Pirabas, Terra Alta e Vigia (Fig. 1). Com exceção de Colares, São João da Ponta e Terra Alta, os demais oito municípios formam a área estudada neste trabalho. De acordo com a classificação de Köppen (Meteoropará 2005), esta área possui tipo climático Am, subtipo Am2. A precipitação pluviométrica média anual varia entre $2.500 \mathrm{~mm}$ e $3.000 \mathrm{~mm}$, tipicamente tropical, caracterizada por duas nítidas estações anuais, uma chuvosa de janeiro a junho e outra menos chuvosa de julho a dezembro. Possui clima megatérmico, com médias anuais máximas entre $30^{\circ}$ e $33^{\circ} \mathrm{C}$, e mínimas entre $21^{\circ} \mathrm{e} 25^{\circ} \mathrm{C}$ (Marinho et al. 2004). Apresenta diferentes tipos de ambientes como campinas, campos, dunas, restingas, capoeiras, igapós, várzeas, manguezais e áreas remanescentes de florestas primárias de terra firme, todas pouco estudadas sob o ponto de vista botânico (Bastos et al. 2001).

\section{Coleta e identificação}

As coletas de briófitas ocorreram em novembro de 1994, abril de 1995 e outubro de 2005 , totalizando 558 amostras. As amostras de briófitas foram coletadas em ecossistemas de mata aberta de terra firme, mata de várzea, mata de igapó, manguezais e capoeiras recentes. O método de coleta foi baseado em Lisboa (1993). As espécies foram classificadas de acordo com os tipos de substrato em que foram coletadas as amostras, segundo Robbins (1952): corticícola - troncos e ramos de árvores vivas; epíxila - ramos e troncos caídos e em decomposição; rupícola - superfície rochosa; terrestre - superfície do solo ou litter. O sistema de classificação adotado neste trabalho é o proposto por Buck \& Goffinet (2000), exceto o da família Leucobryaceae, adotando-se Vitt (1984). O material estudado foi incorporado no 

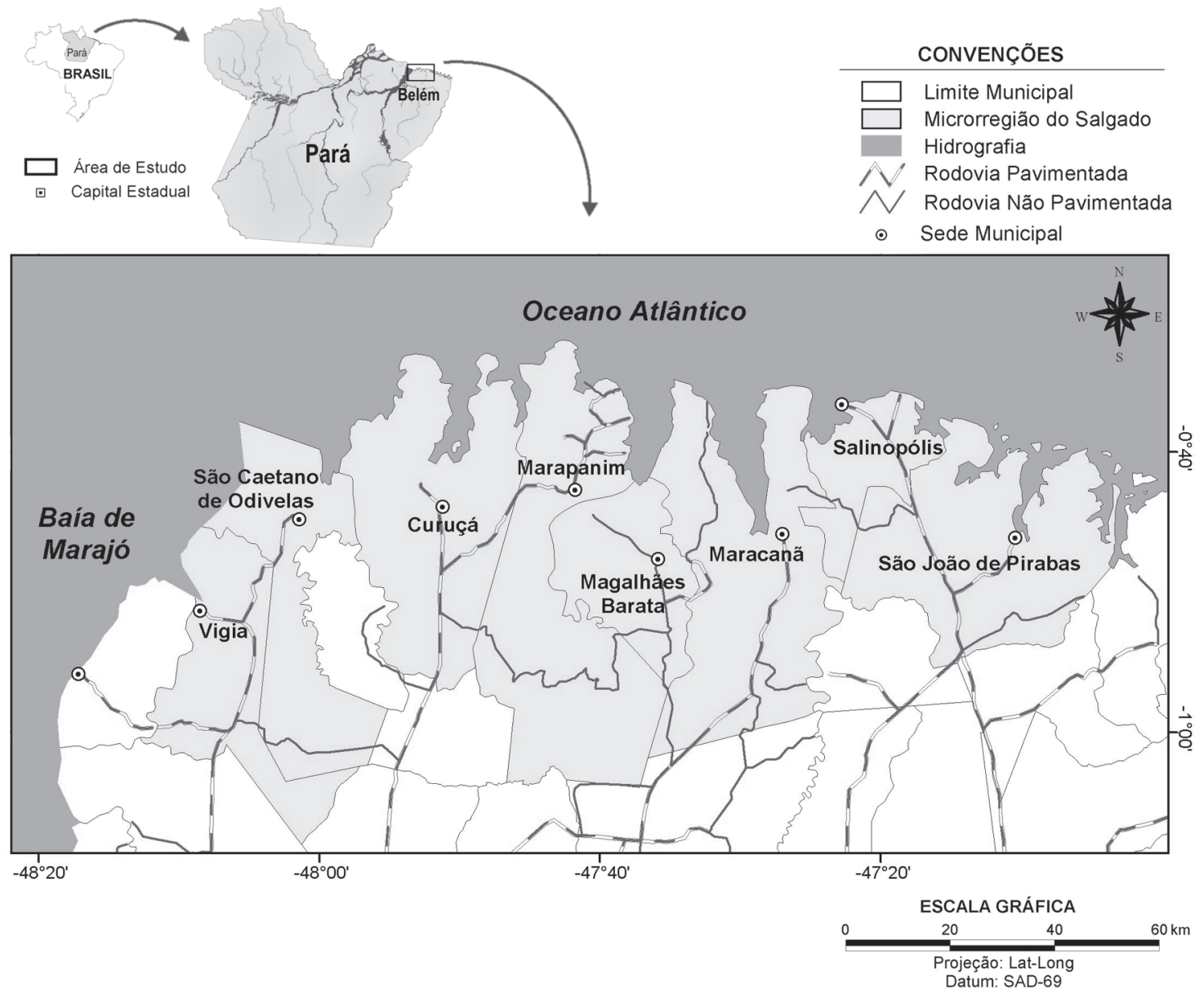

Figura 1 - Localização da microrregião do Salgado-PA, Brasil.

herbário do Museu Paraense Emílio Goeldi (MG), Pará.

\section{Resultados e Discussão}

As 38 espécies de musgos inventariadas na microrregião do Salgado Paraense correspondem a aproximadamente $33 \%$ do número de táxons de musgos que ocorrem no estado do Pará [cerca de 114 táxons de musgos citados por Yano (1981, 1989, 1995, 1996 e 2006)].

Dentre as 558 amostras analisadas, foram observadas 795 ocorrências de musgos nos oito municípios estudados. O número de ocorrências para cada espécie, ecossistema, substrato e município onde foram coletadas encontram-se relacionados na Tabela 1.

Semathophyllaceae, Calymperaceae e Leucobryaceae destacaram-se tanto em diversidade de espécies como em número de ocorrências.
A espécie mais freqüente foi Sematophyllum subsimplex, muito comum na região amazônica (Lisboa \& Ilkiu-Borges 2007; Lisboa \& Illkiu-Borges, F. 1996; Lisboa \& Maciel 1994; Lisboa \& Nazaré 1997; Lisboa et al. 1999; Moraes \& Lisboa 2006; Santos \& Lisboa 2003; Souza \& Lisboa 2005).

Sematophyllum subsimplex, foi coletada em $83,33 \%$ dos ecossistemas estudados e sobre a maioria dos substratos (tab. 1). Muito comum em madeiras em decomposição e base de árvores do sub-bosque em florestas de terra firme; também encontrada em ramos abaixo do dossel e em vegetação seca de cerrado (Florschütz-de Waard \& Velling 1996).

A capoeira apresentou 31 espécies e 336 espécimes e a mata aberta de terra firme $25 \mathrm{e}$ 296, respectivamente (Fig. 2). A riqueza de espécies e número de ocorrências nestes dois ecossistemas superam a somatória das espécies 
e dos espécimes de musgos encontrados na mata de várzea, mata de igapó, restinga e no mangue. Deve ser ressaltado que todos esses números funcionam apenas como indicativos, uma vez que as coletas foram feitas aleatoriamente, sem sistematização para estudos estatísticos.

Segundo Gradstein et al. (2001), as florestas secundárias com maior diversidade florística podem reter de 50-70\% das espécies de briófitas das florestas não perturbadas, ressaltando, portanto, a importância da conservação destes ecossistemas para a sobrevivência dessas espécies.

Quanto aos substratos, Richards (1984) e Germano \& Pôrto (1998), afirmam que nas florestas tropicais úmidas, os troncos vivos seguidos por troncos mortos, são os preferidos para o estabelecimento das briófitas. Isso pode ser observado nos resultados encontrados (Tab. 1), onde os espécimes corticícolos, com 682 ocorrências, superam os epíxilos (103) e terrestres (10). Não foram coletadas espécies rupícolas e epífilas. A ausência de espécies rupícolas explica-se pela pouca disponibilidade de rochas ou pedras nos locais de coleta. Quanto à ausência de espécies epífilas, é explicada por estas serem consideradas de 'sombra' e particularmente vulneráveis a distúrbios no ecossistema (Gradstein 1997), estando entre as primeiras briófitas a desaparecer quando a cobertura das florestas é aberta (Gradstein 1992; Pócs 1996). Scott (1982) ressalta ainda que, em vegetações abertas onde a insolação é intensa, as briófitas são encontradas preferencialmente colonizando casca ou tronco de árvores e arbustos e fendas das rochas, ou seja, em locais onde há alguma proteção contra uma rápida dessecação.

Por ser a microrregião do Salgado Paraense uma zona costeira com influência direta do Oceano Atlântico, alterada naturalmente em função das marés e dos ventos e também pela utilização humana, foram encontradas diversas espécies de musgos típicas de ambientes alterados. Dentre estas, Calymperes afzelii, C. erosum, C. lonchophyllum, C. palisotii, Octoblepharum albidum var. albidum, O. albidum var. violascens, O. pulvinatum, S. subsimplex e Taxithelium planum, são tolerantes a grandes intensidades de luz, altas temperaturas e poluição do ar, o que lhes dá uma amplitude ecológica muito grande (Lisboa \& Ilkiu-Borges 2001; Lisboa \& Ilkiu-Borges F. 1996; Lisboa et al. 1998; Porto 1996; Santos \& Lisboa 2003). Reese (1993) afirma que C. palisotii é particularmente comum em regiões costeiras.

As espécies Calymperes afzelii, $C$. erosum e $C$. palisotii apesar de não serem exclusivas de mangue, foram as únicas encontradas neste ecossistema, confirmando sua tolerância a ambientes litorâneos (Lisboa et al. 1999, Santos \& Lisboa 2003, Visnadi 2004, Costa \& Yano 1998).

Splacnobryum obtusum, comumente encontrada em ambientes perturbados por Lisboa \& Ilkiu-Borges (1997), foi coletada nas mesmas condições na microrregião do Salgado Paraense. Lepidopilum surinamense apresentou-se exclusivamente na mata de igapó e Sematophyllum subpinnatum ocorreu na restinga e capoeira. Estas espécies apresentaram estreita amplitude ecológica.

Entretanto, todas as espécies ocorrentes nos oito municípios desta microrregião estão adaptadas a um complexo de fatores ambientais e encontram as condições necessárias e favoráveis à sua manutenção e reprodução. Com base em todas as observações acima, sugere-se, desta forma, que estas espécies podem ser potencialmente utilizadas como indicadoras de ambientes perturbados.

Estes resultados evidenciam a importância da preservação dos ecossistemas que restam nesta região e desse tipo de estudo para o melhor

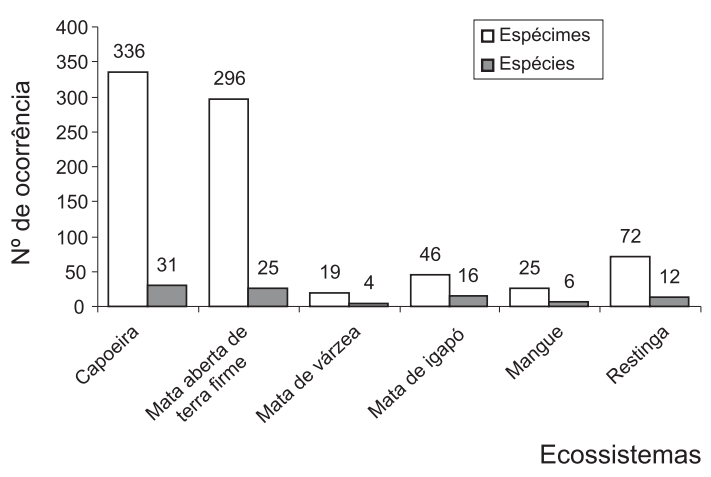

Figura 2-Ocorrência de espécies e espécimes de musgos de acordo com os ecossistemas da microrregião do Salgado Paraense, Brasil. 
Tabela 1 - Musgos dos municípios da microrregião do Salgado Paraense. $\mathrm{N}^{\circ}$ Ocor. - número de ocorrência, F - epífila, C - corticícola, E - epíxila, T - terrestre, $\mathrm{R}$ - rupícola, $\mathrm{CA}$ - capoeira, $\mathrm{TF}$ - mata aberta de terra firme, $\mathrm{MA}$ - mangue, MI - mata de igapó, $\mathrm{RE}$ - restinga, $\mathrm{MV}$ - mata de várzea SAL - Salinópolis, SCO - São Caetano de Odivelas, SJP - São João de Pirabas, CUR - Curuçá, MAP - Marapanim, MAC - Maracanã, MBA - Magalhães Barata, VIG - Vigia.

\begin{tabular}{|c|c|c|c|c|c|c|c|c|c|c|c|c|c|c|c|c|c|c|c|c|}
\hline \multirow[t]{2}{*}{ Família / Espécie } & \multirow[t]{2}{*}{$\begin{array}{l}\mathbf{N}^{\circ} \\
\text { Ocor. }\end{array}$} & \multicolumn{5}{|c|}{ Substrato } & \multicolumn{6}{|c|}{ Ecossistema } & \multicolumn{8}{|c|}{$\begin{array}{l}\text { Municípios da microrregião } \\
\text { do Salgado Paraense }\end{array}$} \\
\hline & & $\mathrm{F}$ & $\mathrm{C}$ & $\mathrm{E}$ & $\mathrm{T}$ & $\mathrm{R}$ & $\mathrm{CA}$ & $\mathrm{TF}$ & MA & MI & $\mathrm{RE}$ & MV & SAL & $\mathrm{SCO}$ & SJP & CUR & MAP & MAC & MBA & VIG \\
\hline \multicolumn{21}{|l|}{ Calymperaceae } \\
\hline Calymperes afzelii $\mathrm{Sw}$. & 11 & & 9 & 2 & & & 6 & 2 & 1 & 1 & 1 & & 3 & 1 & 1 & & 4 & 2 & & \\
\hline Calymperes erosum MüIl. Hal. & 59 & & 51 & 8 & & & 39 & 14 & 1 & 4 & 1 & & 21 & 5 & 16 & 1 & 3 & 4 & 6 & 3 \\
\hline Calymperes lonchophyllum Schwägr. & 32 & & 32 & & & & 1 & 26 & & 1 & & 4 & & 6 & & 11 & & & 11 & 4 \\
\hline Calymperes palisotii Schwägr. & 77 & & 66 & 11 & & & 48 & 4 & 16 & & 9 & & 32 & 14 & & 2 & 21 & 4 & 1 & 3 \\
\hline Calymperes pallidum Mitt. & 8 & & 7 & 1 & & & 4 & 1 & & 3 & & & 2 & & & 1 & 1 & 3 & 1 & \\
\hline Calymperes platyloma Mitt. & 2 & & 2 & & & & & 2 & & & & & & 1 & & 1 & & & & \\
\hline Syrrhopodon cryptocarpus.Dozy \& Molk & 6 & & 6 & & & & & 3 & & 1 & & 2 & 2 & 1 & 1 & & & & & 2 \\
\hline Syrrhopodon incompletus Schwägr. & 6 & & 5 & 1 & & & & 2 & & & & 4 & & 3 & & & & & & 3 \\
\hline Syrrhopodon ligulatus Mont. & 8 & & 8 & & & & 1 & 6 & & & & 1 & 5 & & & 3 & & & & \\
\hline \multicolumn{21}{|l|}{ Dicranaceae } \\
\hline Campylopus surinamensis Müll. Hal. & 8 & & 3 & & 5 & & 7 & 1 & & & & & 1 & & & 3 & 2 & 1 & & 1 \\
\hline $\begin{array}{l}\text { Dicranella hilariana (Mont.) Mitt. } \\
\text { Fissidentaceae }\end{array}$ & 1 & & & & 1 & & 1 & & & & & & & & & & 1 & & & \\
\hline Fissidens elegans Brid. & 2 & & 2 & & & & & 2 & & & & & & 1 & & & & 1 & & \\
\hline $\begin{array}{l}\text { Fissidens guianensis Mont. } \\
\text { Hypnaceae }\end{array}$ & 2 & & 2 & & & & 2 & & & & & & & 2 & & & & & & \\
\hline $\begin{array}{l}\text { Chryso-hypnum diminutivum } \\
\text { (Hampe) W. R. Buck }\end{array}$ & 5 & & 5 & & & & 5 & & & & & & & & & & 4 & & 1 & \\
\hline $\begin{array}{l}\text { Isopterygium subbrevisetum } \\
\text { (Hampe) Broth. }\end{array}$ & 7 & & 6 & 1 & & & 6 & 1 & & & & & 2 & & & 1 & & & 2 & 2 \\
\hline Isopterygium tenerum (Sw.) Mitt. & 2 & & 2 & & & & 2 & & & & & & & & & & 1 & & & 1 \\
\hline $\begin{array}{l}\text { Vesicularia vesicularis (Schwägr.) Broth. } \\
\text { Leucobryaceae }\end{array}$ & 2 & & 2 & & & & 2 & & & & & & & & & & & & 2 & \\
\hline $\begin{array}{l}\text { Leucobryum martianum (Hornsch.) } \\
\text { Hampe ex Müll. Hal. }\end{array}$ & 4 & & 1 & 3 & & & 1 & & & 1 & & 2 & & & & & & & 2 & 2 \\
\hline Ochrobryum gardneri (Müll. Hal.) Mitt. & 7 & & 5 & 2 & & & 3 & 2 & & & & 2 & & & 2 & 1 & & 1 & 1 & 2 \\
\hline Ochrobryum subulatum Hampe & 2 & & 2 & & & & 2 & & & & & & 2 & & & & & & & \\
\hline
\end{tabular}




\begin{tabular}{|c|c|c|c|c|c|c|c|c|c|c|c|c|c|c|c|c|c|c|c|c|}
\hline \multirow[t]{2}{*}{ Família / Espécie } & \multirow[t]{2}{*}{$\begin{array}{l}\mathbf{N}^{\circ} \\
\text { Ocor. }\end{array}$} & \multicolumn{5}{|c|}{ Substrato } & \multicolumn{6}{|c|}{ Ecossistema } & \multicolumn{8}{|c|}{$\begin{array}{l}\text { Municípios da microrregião } \\
\text { do Salgado Paraense }\end{array}$} \\
\hline & & $\mathrm{F}$ & $\mathrm{C}$ & $\mathrm{E}$ & $\mathrm{T}$ & $\mathrm{R}$ & $\mathrm{CA}$ & $\mathrm{TF}$ & MA & MI & $\mathrm{RE}$ & MV & SAL & SCO & SJP & CUR & MAP & MAC & MB A & VIG \\
\hline Octoblepharum albidum Hedw. var. albidum & 59 & & 50 & 7 & 2 & & 35 & 14 & & 1 & 8 & 1 & 22 & 4 & 7 & 6 & 3 & 12 & 1 & 4 \\
\hline $\begin{array}{l}\text { Octoblepharum albidum Hedw. } \\
\text { var violascens Müll. Hal. }\end{array}$ & 27 & & 26 & 1 & & & 14 & 13 & & & & & 12 & 2 & 6 & 5 & & & & 2 \\
\hline $\begin{array}{l}\text { Octoblepharum cylindricum Schimp. } \\
\text { ex Mont. }\end{array}$ & 13 & & 12 & 1 & & & 5 & 5 & & 3 & & & 2 & 4 & & & & 7 & & \\
\hline $\begin{array}{l}\text { Octoblepharum pulvinatum } \\
\text { (Dozy \& Molk.) Mitt. }\end{array}$ & 76 & & 75 & 1 & & & 7 & 52 & & 1 & & 16 & 10 & 33 & & 12 & 2 & 2 & 2 & 15 \\
\hline \multicolumn{21}{|l|}{ Orthotrichaceae } \\
\hline $\begin{array}{l}\text { Groutiella tomentosa (Hornsch.) } \\
\text { Wijk \& Margad. }\end{array}$ & 4 & & 4 & & & & 2 & 2 & & & & & & & & 3 & & & & 1 \\
\hline \multicolumn{21}{|l|}{ Pilotrichaceae } \\
\hline $\begin{array}{l}\text { Callicostella pallida (Hornsch.) } \\
\text { Ångström }\end{array}$ & 3 & & 2 & 1 & & & 1 & & & 2 & & & & & & 1 & & & 2 & \\
\hline $\begin{array}{l}\text { Lepidopilum surinamense Müll. Hal. } \\
\text { Sematophyllaceae }\end{array}$ & 1 & & 1 & & & & & & & 1 & & & & & & & & & 1 & \\
\hline $\begin{array}{l}\text { Sematophyllum subpinnatum } \\
\text { (Brid.) E. Britton }\end{array}$ & 2 & & 1 & 1 & & & 1 & & & & 1 & & 1 & & & & & & 1 & \\
\hline Sematophyllum subsimplex (Hedw.) Mitt. & 278 & & 232 & 45 & 1 & & 114 & 108 & & 21 & 5 & 30 & 84 & 41 & 36 & 18 & 7 & 39 & 24 & 29 \\
\hline Taxithelium planum (Brid.) Mitt. & 21 & & 15 & 6 & & & 10 & 6 & & 2 & & 3 & & 1 & 3 & 5 & 3 & 1 & 5 & 3 \\
\hline Trichosteleum intricatum (Thér.) J. Florsch. & 14 & & 13 & 1 & & & & 10 & 1 & 1 & & 2 & 5 & 5 & & 1 & & & 1 & 2 \\
\hline $\begin{array}{l}\text { Trichosteleum papillosum (Hornsch.) } \\
\text { A. Jaeger }\end{array}$ & 13 & & 12 & 1 & & & 6 & 5 & & 2 & & & 9 & 1 & & 1 & 1 & 1 & & \\
\hline $\begin{array}{l}\text { Trichosteleum subdemissum } \\
\quad \text { (Schimp. ex Besch.) A. Jaeger }\end{array}$ & 6 & & 5 & 1 & & & 5 & & & 1 & & & 2 & & & & 3 & & 1 & \\
\hline \multicolumn{21}{|l|}{ Splachnobryaceae } \\
\hline $\begin{array}{l}\text { Splachnobryum obtusum (Brid.) } \\
\text { Müll. Hal. }\end{array}$ & 1 & & & & 1 & & 1 & & & & & & & & & & 1 & & & \\
\hline \multicolumn{21}{|l|}{ Stereophyllaceae } \\
\hline $\begin{array}{l}\text { Entodontopsis leucostega (Brid.) } \\
\text { W.R. Buck \& Ireland }\end{array}$ & 1 & & & 1 & & & 1 & & & & & & 1 & & & & & & & \\
\hline $\begin{array}{l}\text { Pilosium chlorophyllum (Hornsch.) } \\
\text { Müll. Hal. }\end{array}$ & 21 & & 14 & 7 & & & 3 & 12 & & & & 6 & 3 & 9 & & 2 & 3 & 1 & & 3 \\
\hline \multicolumn{21}{|l|}{ Thuidiaceae } \\
\hline Pelekium scabrosulum (Mitt.) Touw & 2 & & 2 & & & & 1 & 1 & & & & & & & & 1 & 1 & & & \\
\hline Total & 795 & & 682 & 103 & 10 & & 336 & 296 & 19 & 46 & 25 & 73 & 221 & 135 & 72 & 80 & 61 & 79 & 64 & 82 \\
\hline
\end{tabular}


conhecimento da brioflora do Pará, alertando para o perigo do desaparecimento de espécies com a destruição das florestas primárias.

\section{Agradecimentos}

À Dra. Anna Luíza Ilkiu-Borges pela tradução do resumo para o inglês; ao Marcelo Thales pela confecção do mapa da microrregião do Salgado Paraense e ao CNPq pela concessão da bolsa de mestrado à primeira autora.

\section{REFERÊNCIAS BIBLIOGRÁFICAS}

Bastos, M. N. C.; Santos, J. U. M.; Amaral, D. D. \& Neto, S. V.C. 2001. Alterações ambientais na vegetação litorânea do nordeste do Pará. In: Prost, M. T.; Mendes, A. C. (ed.). Ecossistemas costeiros: impactos e gestão ambiental. MPEG, Belém. Pp. 28-38.

Buck, W. R. \& Goffinet, B. 2000. Morphology and classification of mosses. In: Shaw, A. J. \& Goffinet, B. (eds.). Bryophyte biology. Vol. 3. University Press, Cambridge. Pp. 71-123.

Costa, D. P. \& Yano, O. 1998. Briófitas da Restinga de Macaé, Rio de Janeiro, Brasil. Hoehnea 25(2): 99-119.

Frahm, J.-P. \& Kirchhoff, K. 2002. Antifeeding effects of bryophyte extracts from Neckera crispa and Porella obtusata against the slug Arion lusitanicus. Cryptogamie, Bryologie 23(3): 271-275.

Germano, S. R. \& Pôrto, K. C. 1998. Briófitas epíxilas de uma área remanescente de Floresta Atlântica (Timbaúba-PE, Brasil). Acta Botanica Brasilica 3(1): 53-66.

Gradstein, S. R. 1992. The vanishing tropical rain forest. As environment for bryophytes and lichens. In: Bates, J. W.; Farmer, A. M. (ed.). Bryophytes and lichens in a chaging environment. Claredon Press, Oxford. Pp. 234-258.

1997. The taxonomic diversity of Epiphyllous Bryophytes. Abstracta Botanica 21 (1): 15-19.

; Churchill, S. P. \& Salazar-Allen, N. 2001. Guide to the bryophytes of tropical America. Memoirs of The New York Botanical Garden 86: 1-577.
Lisboa, R. C. L. 1993. Musgos acrocárpicos do estado de Rondônia. Museu Paraense Emílio Goeldi, Belém, 272p.

\& Ilkiu-Borges, A. L. 1995. Diversidade das Briófitas de Belém (PA) e seu potencial como indicadoras de poluição urbana. Boletim do Museu Paraense Emílio Goeldi, Série Botânica 11(2): 199-225. \& Ilkiu-Borges, A. L. 1997. A família Splachnobryaceae (Bryophyta) no estado do Pará. Boletim do Museu Paraense Emílio Goeldi, Série Botânica 13(2): 103-111. \& Ilkiu-Borges, A. L. 2001. Briófitas de São Luís do Tapajós, município de Itaituba, com novas adições para o estado do Pará. Boletim do Museu Paraense Emílio Goeldi, Série Botânica 17(1): 75-91. \& Ilkiu-Borges, A. L. 2007. Uma nova avaliação da brioflora da Reserva do Mocambo, Belém (PA) In: Gomes, J. I. (org.). Mocambo: diversidade e dinâmica biológica da Área de Pesquisa Ecológica do Guamá (APEG). Museu Paraense Emílio Goeldi, Belém. Pp. 149-174. \& Ilkiu-Borges, F. 1996. Briófitas da Serra dos Carajás e sua possível utilização como indicadoras de metais. Boletim do Museu Paraense Emílio Goeldi, série Botânica 12(2): 161-181. ; Lima, M. J. L. \& Maciel, U. N. 1999. Musgos da Ilha de Marajó-II- Anajás Pará, Brasil. Acta Amazonica 29(2): 201-206. \& Maciel, U. N. 1994. Musgos da Ilha de Marajó-I-Afuá, Pará. Boletim Museu Paraense Emílio Goeldi, série Botânica 10(1): 43-56. ; Muniz, A. C. M. \& Maciel, U. N. 1998. Musgos da Ilha de Marajó-IIIChaves (Pará). Boletim do Museu Paraense Emílio Goeldi, série Botânica 14(2): 117-125.

\& Nazaré, J. M. M. de. 1997. A flora briológica. In: Lisboa, P.L. B.(org.). Caxiuanã. Vol. 4. CNPq/MPEG, Belém. Pp. 223-235.

Marinho, R. S; Ferreira, A. L. O.; Costa, B. M. N. \& Barbosa, E. J. S. 2004. Aspectos geográficos da zona costeira paraense: o caso do município de Salinópolis. VICNBG. UERJ. Disponível 
em <:http://www.igeo.uerj.br/VICBG 2004/ Eixo2/E2_112.htm>. Acesso em: 1/XI/2005.

Meteoropará, Classificação Climática do Pará (Método de Köppen). Disponível em: <http:/www.meteoropara.hpg.ig.com.br/ matdidatico/classificacao.htm $>$. Acessado em: 29 de outubro de 2005. 29/X/2005.

Moraes, E. N. R. \& Lisboa, R. C. L. 2006. Inventário dos musgos (Bryophyta) da Serra dos Carajás, estado do Pará. Boletim do Museu Paraense Emílio Goeldi, série Ciências Naturais 2(1): 39-68.

Pócs, T. 1996. Epiphyllous liverworts diversity at worldwide level and its threat and conservation. Anales Instituto Biologia Universidade Nacional Autónoma México, Série Botânica 67(1): 109-127.

Pôrto, K. C. 1996. Briófitas. In: Sampaio, E. V. S. B.; Mayo, S. J. \& Barbosa, M. R. V. (eds.). Pesquisa botânica nordestina: progresso e perspectivas. Sociedade Botânica do Brasil/Seção Regional de Pernambuco, Recife. Pp. 97-109.

Rao, D. N. 1982. Bryophyte ecology. In: Smitth, A. G. E. (ed.). Responses of bryophytes to air pollution. New York. Pp. 445-472.

Reese, W. D. 1993. Calymperaceae. Flora Neotropica Monograph 58: 1-102.

Richards, P. W. 1984. The Ecology of Tropical Forest Bryophytes. In: Schuster, R. M. New Manual of Bryology, Nichinan: The Hattori Botanical Laboratory 2: 1233-1269.

Robbins, R. G. 1952. Bryophyta ecology of a dune area in New Zealand. Vegetation, Acta Geobotanica 4: 1-131.

Salomão, R. de P.; Nepstad, D. C. \& Vieira, I. C. G. 1996. Como a biomassa de florestas tropicais influi no efeito estufa? Ciência Hoje 21(123): 38-47.

Santos, R. C. P \& Lisboa, R. C. L. 2003. Musgos (Bryophyta) do nordeste paraense, Brasil - 1. Zona Bragantina, microrregião do Salgado e município de Viseu. Acta Amazonica 33(3): 415- 422.

Scott, G. A. M. 1982. Desert Bryophytes. In: Smith, A.J.E. (ed.). Bryophyte Ecology.
Chapman \& Hall, London. Pp.105-122.

SEPOF - Secretaria Executiva de Estado de Planejamento, Orçamento e Finanças. 2005. Estatística Municipal - microrregião do Salgado. Governo do Estado do Pará, Belém. Disponível em: <http://www.sepof.pa.gov.br/ microrregiao.cfm\#salgado $>$ Acesso em: 14/I/2006.

Sergio, C. \& Bento-Pereira, F. 1981. Liquenes e briófitos como bioindicadores da poluição atmosférica. Boletim da Sociedade Broteriana, Ser. 2, 54: 291-303.

Shaw, A. J. \& Goffinet, B. (eds.). 2000. Bryophyte biology. Cambridge University Press, Cambridge, 476p.

Souza A. P. S. \& Lisboa R. C. L. 2005. Musgos (Bryophyta) na ilha Trambioca, Barcarena, PA. Acta Botanica Brasilica 19(3): 487-492.

Visnadi, S. R. 2004. Briófitas de praias do estado de São Paulo, Brasil. Acta Botânica Brasilica 18(1): 91-97.

Vitt, D. H. 1984. Classification of the Bryopsida. In: Shuster, R. W. (ed.). New manual of bryology. Nichinan. The Journal Hattori Botanical Laboratory 2: 696-759.

Wagner, D. K. 1995. Mesos e microrregiões formam um grande estado. Nosso Pará 2: 12-13.

Yano, O. 1981. A checklist of Brasilian mosses. The Journal Hattori Botanical Laboratory 50: 279-456.

1989. An additional checklist of Brazilian Bryophytes. Journal Hattori Botanical Laboratory 66: 371-434. 1995. A new additional checklist of Brazilian Bryophytes. The Journal Hattori Botanical Laboratory 78: 137-182. .1996. A checklist of Brazilian Bryophytes. Boletim do Instituto de Botânica 10: 47-232. .2006. Novas adições ao catálogo de briófitas brasileiras. Boletim do Instituto de Botânica 17: 1-42.

Zartman, C. E. 2003. Habitat fragmentation impacts on epiphyllous bryophyte communities in central amazonia. Ecology 84(4): 948-954. 